\title{
Aggressive strategy to save the brain in a case of acute aortic dissection
}

\author{
Kenji Minatoya ${ }^{1}$ \\ ${ }^{1}$ Kyoto Daigaku
}

July 22, 2021

\begin{abstract}
The case report by Sicim et al. is the placement of extra-anatomical bypasses in bilateral common carotid arteries. The similar previous reports of the extra-anatomical bypass usually indicate unilateral bypass. Whether or not the Willis' circle is incomplete is difficult to judge during emergency surgery, and the authors' judgment seems to have been correct in the sense that it could maintain cerebral perfusion reliably and quickly. The direct perfusion and extraanatomical bypass of carotid artery is a reasonable strategy in patients with cerebral malperfusion.
\end{abstract}

Title: Aggressive strategy to save the brain in a case of acute aortic dissection

Kenji Minatoya, MD, PhD

Professor and Chairman

Department of Cardiovascular Surgery,

Graduate School of Medicine, Kyoto University,

Kyoto, Japan

Word count: 927

Abstract

The case report by Sicim et al. is the placement of extra-anatomical bypasses in bilateral common carotid arteries. The similar previous reports of the extra-anatomical bypass usually indicate unilateral bypass. Whether or not the Willis' circle is incomplete is difficult to judge during emergency surgery, and the authors' judgment seems to have been correct in the sense that it could maintain cerebral perfusion reliably and quickly. The direct perfusion and extraanatomical bypass of carotid artery is a reasonable strategy in patients with cerebral malperfusion.

Cerebral malperfusion as a complication of acute aortic dissection is a predictor of a poor prognosis. [1][2][3] The malperfusion in acute aortic dissection is majorly induced by true lumen compression by a false lumen or intimal flap, but it is not always relieved by so-called central repair such as ascending aortic replacement. Therefore, it is always necessary to decide whether to give priority to the central repair or to release the malperfusion directly depending on the situation. The central repair may be prioritized if neurological symptoms such as loss of consciousness after onset are temporary, but if some neurological symptoms persist, rapid measures for reperfusion of the brain must be considered.

This issue of this journal features a case report by Sicim et al. describing direct bilateral carotid artery cannulations in a case with extended dissection to the carotid arteries. [4] The patient had vision loss in both eyes and monoplegia in the left arm. They firstly commenced direct bilateral carotid artery perfusion, 
then established the extracorporeal circulation. Although it took some time, the patient finally recovered to a state without neurological abnormalities after rehabilitation. I would like to commend the devoted efforts of the entire team, including not only this surgical strategy, but also the fact that this rapid cerebral reperfusion was started in an extremely short time of less than 30 minutes from the time of the hospital visit.

As a method for releasing the cerebral malperfusion, it is important to secure the aortic return of the cardiopulmonary bypass in the true lumen. Shimura et al. reported that the neurological symptoms of patients with preoperative cerebral malperfusion were alleviated by performing the secure true lumen cannulation at the ascending aorta using the Seldinger technique. [5]

Femoral artery and axillary artery have been popular cites as the aortic return among the peripheral arteries. [6] Yet, axillary artery, subclavian artery, and innominate artery are better to maintain the antegrade flow and they might improve the cerebral malperfusion since they are close to the arch branches. [7] The carotid artery is known to be more accessible than the axillary artery and has less risk of nerve damage. Urbanski et al. utilized the carotid artery as a site of aortic return through a short graft anastomosed to the end side instead of direct cannulation. [8] In addition, multiple cannulations might be another option to restore true lumen flow and relieve the malperfusion. [9]

Selective cerebral perfusion (SCP) in aortic surgery is an established brain protection method. The standard method is to perfuse brachiocephalic artery, left common carotid artery, and left subclavian artery, but it has been reported that it is also possible to perfuse only the brachiocephalic artery.[10] The SCP from only one side functions safely as brain protection in most cases, but considering safety, perfusion from all branches of the aortic arch is recommended because there are many cases where the Willis' ring is incomplete in the skull. [11] Moreover, as in this case, when the branches of the arch are dissected, the brain protection is incomplete by the standard method of selective cerebral perfusion from only the orifice of the arch branches. In such cases, it makes sense to secure a blood supply channel directly to the periphery of the arch branch.

Several cases regarding this extra-anatomical bypass to neck arteries have been reported. Gomibuchi et al. reported that postoperative neurological complications were reduced by additional cannulation at the common carotid artery in patients with preoperative imaging cerebral malperfusion. [12] Sugiyama et al. performed direct cannulation to the common carotid artery in addition to the usual selective cerebral perfusion to all branches of the aortic arch for cases in which dissection had extended to the common carotid artery on preoperative CT imaging in their later series. They described the neurological complications have been drastically reduced with this aggressive method. [13] The mechanism of the cerebral malperfusion is not always same, however, when a major entry is in the ascending aorta, malperfusion mostly occurs on the right due to the location of the entry and a unique configuration of vessel bifurcation. The right common carotid artery is obstructed by either the intimal flap in the innominate artery or by an expanded false lumen. [14] What is interesting in this case report by Sicim et al. is the placement of extra-anatomical bypasses in bilateral common carotid arteries. Whether or not the Willis' circle is incomplete is difficult to judge during emergency surgery, and the authors' judgment seems to have been correct in the sense that it could maintain cerebral perfusion reliably and quickly.

Okita et al. have introduced a method for maintaining cerebral perfusion using a shunt from the femoral artery to the right common carotid artery preoperatively. [15] They described their criteria of the direct cannulation of the carotid artery. They are at least one sign or symptom of diminished flow in the carotid arteries, such as dissected carotid arteries with compressed true lumen by CT scan, decreased blood flow in the carotid artery demonstrated by ultrasound, persistent consciousness disorder, hemiparesis, or tight to left difference in forehead rSO2 or arm blood pressures. However, it has also been pointed out that it is necessary to control the perfusion rate for cerebral ischemia to prevent reperfusion injury. [16]

To further improve the surgical results of acute aortic dissection, it is important to establish a strategy for cerebral malperfusion. Once again, I would like to congratulate the authors saving the patient without severe neurological symptoms by adopting the aggressive strategy, and clarify that this case report will be useful 
information for our readers.

[1] Pansini S, Gagliardotto PV, Pompei E, Parisi F, Bardi G, Castenetto E, Orzan F, di Summa M. Early and late risk factors in surgical treatment of acute type A aortic dissection

Ann Thorac Surg. 1998 Sep;66(3):779-84.

[2] Tanaka H, Okada K, Yamashita T, Morimoto Y, Kawanishi Y, Okita Y. Surgical results of acute aortic dissection complicated with cerebral malperfusion. Ann Thorac Surg. 2005 Jul;80(1):72-6.

[3] Czerny M, Schoenhoff F, Etz C, Englberger L, Khaladj N, Zierer A, Weigang E, Hoffmann I, Blettner M, Carrel TP. The Impact of Pre-Operative Malperfusion on Outcome in Acute Type A Aortic Dissection: Results From the GERAADA Registry.

J Am Coll Cardiol. 2015 Jun 23;65(24):2628-2635.

[4] Sicim H, Demirdas E, Bolcal C. Direct bilateral carotid artery cannulation can be better strategy for acute aortic dissection involving both carotid arteries. J Card Surg. in press

[5] Shimura S, Odagiri S, Furuya H, Okada K, Ozawa K, Nagase H, Yamaguchi M, Cho Y. Echocardiographyguided aortic cannulation by the Seldinger technique for type A dissection with cerebral malperfusion. J Thorac Cardiovasc Surg. 2020 Mar;159(3):784-793.

[6] Sabik JF, Lytle BW, McCarthy PM, Cosgrove DM. Axillary artery: an alternative site of arterial cannulation for patients with extensive aortic and peripheral vascular disease. J Thorac Cardiovasc Surg. 1995 May;109(5):885-90; discussion 890-1.

[7] Rylski B, Urbanski PP, Siepe M, Beyersdorf F, Bachet J, Gleason TG, Bavaria JE. Operative techniques in patients with type A dissection complicated by cerebral malperfusion. Eur J Cardiothorac Surg. 2014 Aug;46(2):156-66.

[8] Urbanski PP. Carotid artery cannulation in acute aortic dissection with malperfusion.

J Thorac Cardiovasc Surg. 2006 Jun;131(6):1398-9.

[9] Minatoya K, Ogino H, Matsuda H, Sasaki H. Rapid and safe establishment of cardiopulmonary bypass in repair of acute aortic dissection: improved results with double cannulation. Interact Cardiovasc Thorac Surg. 2008 Dec;7(6):951-3.

[10] Norton EL, Wu X, Kim KM, Patel HJ, Deeb GM, Yang B.

Unilateral is comparable to bilateral antegrade cerebral perfusion in acute type A aortic dissection repair. J Thorac Cardiovasc Surg. 2020 Sep;160(3):617-625.

[11] Papantchev V, Stoinova V, Aleksandrov A, Todorova-Papantcheva D, Hristov S, Petkov D, Nachev G, Ovtscharoff $\mathrm{W}$. The role of Willis circle variations during unilateral selective cerebral perfusion: a study of 500 circles. Eur J Cardiothorac Surg. 2013 Oct;44(4):743-53.

[12] Gomibuchi T, Seto T, Naito K, Chino S, Mikoshiba T, Komatsu M, Tanaka H, Ichimura H, Yamamoto T, Nakahara K, Ohashi N, Fuke M, Wada Y, Okada K. Strategies to improve outcomes for acute type A aortic dissection with cerebral malperfusion. Eur J Cardiothorac Surg. 2021 Apr 13;59(3):666-673.

[13] Sugiyama K, Watanuki H, Futamura Y, Okada M, Oiwa T, Makino S, Matsuyama K. Impact of direct carotid artery perfusion in acute type A aortic dissection involving the common carotid artery. Gen Thorac Cardiovasc Surg. 2021 Apr 2:1-9.

[14] Orihashi K. Cerebral malperfusion in acute aortic dissection. Surg Today. 2016 Dec;46(12):1353-1361.

[15] Okita Y, Ikeno Y, Yokawa K, Koda Y, Henmi S, Gotake Y, Nakai H, Matsueda T, Inoue T, Tanaka H. Direct perfusion of the carotid artery in patients with brain malperfusion secondary to acute aortic dissection. Gen Thorac Cardiovasc Surg. 2019 Jan;67(1):161-167. 
[16] Munakata H, Okada K, Kano H, Izumi S, Hino Y, Matsumori M, Okita Y. Controlled earlier reperfusion for brain ischemia caused by acute type A aortic dissection. Ann Thorac Surg. 2009 Apr;87(4):e27-8. 